\title{
Full Mouth Rehabilitation of a Patient with Severe Attrition Using Digital Smile Design Concept: Case Report
}

\author{
Ahmed A Elsayyad* \\ Department of Prosthetic Dentistry, Faculty of Oral and Dental Medicine, Cairo University, Egypt \\ *Corresponding author: Ahmed A Elsayyad, Department of Prosthetic Dentistry, Faculty of Oral and Dental Medicine, \\ Cairo University, Egypt
}

\section{ARTICLE INFO}

Received: 慧 March 09, 2020

Published: 䋓 March 18, 2020

Citation: Ahmed A E. Full Mouth Rehabilitation of a Patient with Severe Attrition Using Digital Smile Design Concept: Case Report. Biomed J Sci \& Tech Res 26(4)-2020. BJSTR. MS.ID.004390.

\begin{abstract}
Prosthetic treatment of patients with severe wear is challenging task. First step is controlling the causative factor that led to teeth wear along with the restoration of lost dentition. This case report describes the full mouth rehabilitation of a patient with severe attrition. Facebow and centric relation records were used to mount maxillary and mandibular models respectively. Over-erupted teeth beyond the occlusal plane of the mounted models were extracted. Diagnostic waxing-up guided by digital smile design was used in treatment planning, based on which silicone keys were constructed. Direct composite resin was used to rebuild the teeth, using silicone keys, for both vertical dimension increase and interim restorative phases. Composite restorations guided by digital smile design can serve as an excellent tool for vertical dimension increase when compared to removable occlusal splint in terms of better patients' adaptation, esthetics and masticatory performance.
\end{abstract}

Keywords: Full Mouth Rehabilitation; Vertical Dimension Increase; Attrition; Teeth Wear; Digital Smile Design

\section{Introduction}

Full mouth rehabilitation of patients with severe attrition represents tremendous challenge. The first issue to consider in treatment planning is addressing the cause. Muts et al. conducted a systematic review on the available treatment options for teeth wear dividing them into three phases: Vertical Dimension Increase (VDI), interim and definitive restorative phases [1]. Mehta et al. used occlusal splint and composite resin for VDI and the interim phases respectively [2]. For the rehabilitation of patient with severe erosion, Schwarz et al. used direct composite for both VDI and interim phases [3]. This can be advantageous in terms of reducing treatment time and cost and consequently greater patient satisfaction when compared to using removable occlusal splint for VDI [3-5]. Digital Smile Design (DSD) has served as a powerful tool for executing and explaining to the patient the proposed treatment plan. It can also be time saving in terms of decreasing the need for in tra-oral mockup where the patient can visualize the expected esthetic outcome on the computer software. The present case report aimed at full-mouth rehabilitation of a patient with severe attrition using direct composite resin in the VDI and interim phases to simplify the treatment plan. DSD was used as a guide during waxing up along with silicone keys for accurate transfer of the desired plan.

\section{Case Presentation}

A 54-year-old, male patient was referred to the Prosthodontics department of our School of Dentistry complaining of disability to eat. Ethics committee approval as well as patient's informed consent was obtained to include his photographs and radiographs in our study. The patient had previous history of angina pectoris and is currently on anticoagulants therapy. He had previous history of bruxism with multiple missing teeth due to caries. Extra-oral 
examination revealed loss of the vertical dimension of occlusion. Temporomandibular joint and muscles of mastication were asymptomatic with no functional limitation. Photographs of different intra-oral views were captured (Figure 1). Furthermore, panoramic and full mouth peri-apical radiographs were requested. Maxillary and mandibular impressions were recorded. Maxillary model was mounted using face bow record. Mandibular models were mounted twice: in centric relation at the accepted raised vertical dimension with two mm freeway space (Figure 2a) and maximum intercuspation. Occlusal plane analysis showed that tooth \#31 is far beyond the occlusal plane. DSD, employing $75 \%$ width to length ratio, was done on Microsoft power point template using functional video to determine the high and low smile lines (Figure $2 \mathrm{~b}$ ). The patient rejected doing crown lengthening or implant surgery because of his medical condition. Silicone keys were constructed based on the waxed-up models (Figure 2c) to transfer the proposed treatment plan intra-orally.

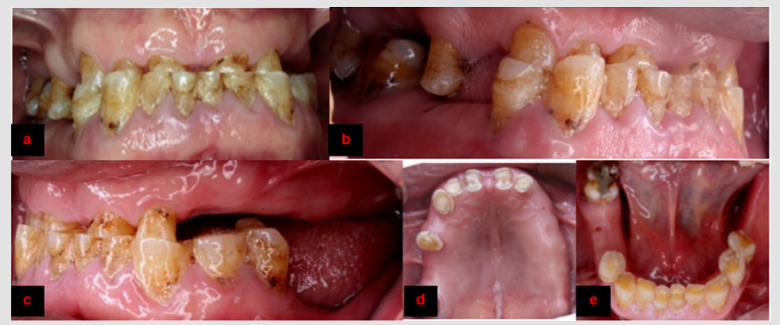

Figure 1:

a. Frontal view showing edge to edge anterior teeth position.

b. Right side showing severe teeth attrition.

c. Left side showing loss of inter-arch space.

d. Maxillary view showing exposed pulp horns of anterior teeth.

e. Mandibular view showing exposed pulp horns of central incisors.

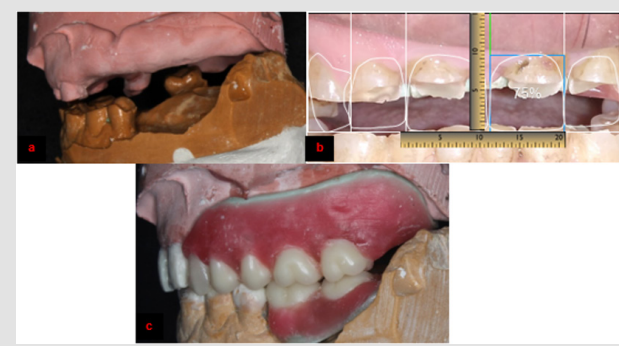

Figure 2:

a. Mounted diagnostic casts at accepted raised vertical dimension.

b. DSD used as a guide during waxing up. Tooth\# 10 is longer cervically than proposed DSD and contralateral one.

c. Posterior interarch space is now available on the waxed-up model.

Teeth \#6, 7, 8, 9, 10, 25 were endodontically treated due to clinical exposure of their pulp horns (Figure 3a). Teeth \#24, 31 were extracted because of grade III mobility and severe over-eruption respectively three weeks before temporization. Maxillary anterior teeth were then restored with fiber posts (Radix®, Dentsply Maillefer, Switzerland) and dual cured composite restorations (Charm Core $\AA^{\circledR}$, Dentkist, Korea) using silicone keys. Mandibular teeth and edentulous spaces were restored with composite resin and acrylic removable partial denture respectively (Figure $3 \mathrm{~b}$ ). Throughout two months follow-up period, the patient reported extreme satisfaction and no discomfort to the raised vertical dimension. Occlusal splint was used during and after temporization as well as definitive phases to control bruxism. In the definitive phase, teeth \# 2, 4, 6, 7, 8, 9, 10 received Porcelain Fused to Metal (PFM) crowns for protection of endodontically treated teeth and occlusal plane refinement (Figure $4 \mathrm{a} \& 4 \mathrm{~b}$ ). Partial dentures with esthetic clasps (iFlex ${ }^{\mathrm{TM}}$, TCS, USA), as requested by the patient, were constructed. The patient reported satisfaction throughout six months follow-up period.

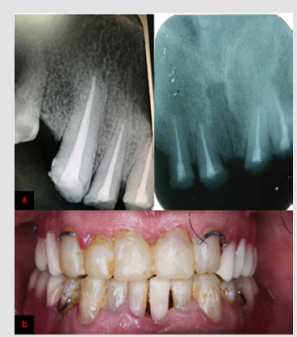

Figure 3:

a. Peri-apical radiographs showing endodontic treatment of maxillary anterior teeth.

b. Silicone keys used to restore maxillary and mandibular teeth according to the proposed waxing-up.

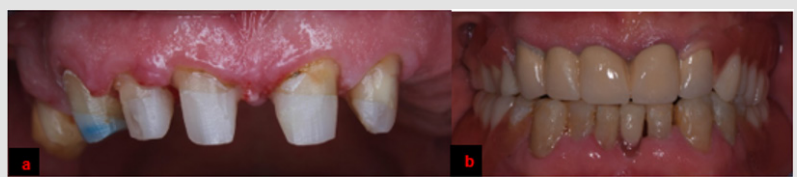

Figure 4:

a. Maxillary teeth were reduced.

b. Final esthetic outcome. Tooth \# 10 is longer than \# 7 which will be masked by the pink colored clasp of the removable partial denture mimicking gingiva to avoid the need for periodontal surgery.

\section{Discussion}

This case report employed direct composite resin in the VDI and interim phases as well as DSD for the rehabilitation of patient with severe attrition. Muts et al. concluded that composite resin is the restoration of choice in the temporization phase [1]. Abduo et al. concluded that permanent VDI is safe and predictable with better patients' adaptation to fixed rather than removable prostheses [6]. Increasing the occlusal vertical dimension up to five $\mathrm{mm}$ is well tolerated by patients with self-limiting signs and symptoms [6]. These findings, in contrast to the workflow suggested by Me- 
hta et al. [2], encourage the use of composite resin for VDI as an alternative to occlusal splint. Although DSD was employed in our study, an entirely digital workflow using 3D DSD, 3D printed digitally waxed up models may have improved the results [7-9]. Cattoni et al. suggested that merging extra-oral and intra-oral photographs of the patient with the STL file of the digital intra-oral impression or the scanned articulated models can convert 2D into 3D-DSD [7]. 3D-DSD approach can be used in the fabrication of waxed up articulated models, provisional restorations or indirect composite restorations $[8,10]$. Another limitation of our study is the short-term follow-up period.

\section{Conclusion}

Direct composite resin may be very promising for VDI instead of occlusal splint, in patients with severe wear, in terms of decreasing treatment time and cost and more accurate testing of the raised occlusal vertical dimension. DSD can be of great help for either analogue or digital waxing-up as well as powerful demonstrating tool to the patient. Well-designed clinical trials are required to validate these findings.

\section{Ethical Statement}

1. Funding: (if any): No sources of funding were available to conduct this study.

2. Conflict of Interest: The authors declare that there is no conflict of interest.

3. Ethical Approval: Approval by the Ethics committee was obtained for the conduct of this study.

4. Informed Consent: Patient's informed consent was obtained to include his photographs and radiographs in our study.

\section{Authors' Contribution}

Both authors (A.E, N.A):

1. Have made substantial contributions to conception and design of the study.

\section{ISSN: 2574-1241}

DOI: $10.26717 /$ BJSTR.2020.26.004390

Ahmed A Elsayyad. Biomed J Sci \& Tech Res

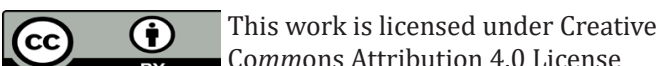

Submission Link: https://biomedres.us/submit-manuscript.php
2. Have drafted the article and revised it critically.

3. Have approved the final version to be published.

\section{References}

1. Muts EJ, van Pelt H, Edelhoff D, Krejci I, Cune M (2014) Tooth wear: A systematic review of treatment options. J Prosthet Dent 112(4): 752759.

2. Mehta SB, Banerji S, Millar BJ, Suarez-Feito JM (2012) Current concepts on the management of tooth wear. Part III: Active restorative care 2: The management of generalised tooth wear. Br Dent J 212(3): 121-127.

3. Schwarz S, Kreuter A, Rammelsberg P (2011) Efficient prosthodontic treatment in a young patient with long-standing bulimia nervosa: A clinical report. J Prosthet Dent 106(1): 6-11.

4. Vailati F, Belser UC (2011) Palatal and facial veneers to treat severe dental erosion: A case report following the three-step technique and the sandwich approach. Eur J Esthet Dent 6(3): 268-278.

5. Grütter L, Vailati F (2013) Full-mouth adhesive rehabilitation in case of severe dental erosion, a minimally invasive approach following the 3-step technique. Eur J Esthet Dent 8(3): 358-375.

6. Abduo J (2012) Safety of increasing vertical dimension of occlusion: A systematic review. Quintessence Int 43(5): 369-380.

7. Cattoni F, Mastrangelo F, Gherlone EF, Gastaldi G (2016) A new total digital smile planning technique (3D-DSP) to fabricate CAD-CAM mockups for esthetic crowns and veneers. Int J Dent 2016: 1-5.

8. Brenes C, Babb CS, Bencharit S, Romero M, Arce R (2017) Digital approach to the fabrication of a wax prototype for full-mouth rehabilitation of a worn dentition: A clinical report. J Oral Science Rehabilitation 3(4): 4247.

9. Daher R, Ardu S, Vjero O, Krejci I (2018) 3D Digital Smile Design With a Mobile Phone and Intraoral Optical Scanner. Compend Contin Educ Dent 39(6): 5-8.

10. Mizrahi B (2008) Combining traditional and adhesive dentistry to reconstruct the excessively worn dentition. Eur J Esthet Dent 3(3): 270289.

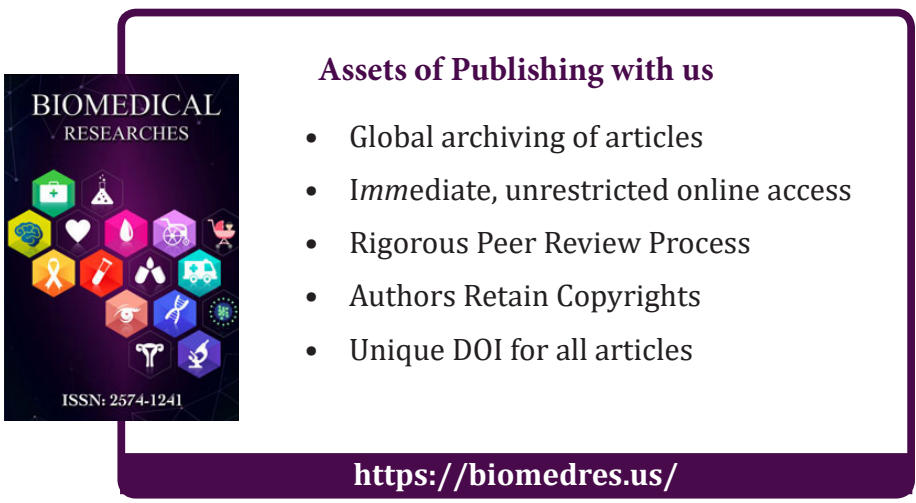

Copyright@ Ahmed A Elsayyad | Biomed J Sci \& Tech Res | BJSTR. MS.ID.004390. 